\title{
Clinical-Homeopathic Profile in the Pediatric Ward at the University Hospital - Brazil
}

\section{Débora Alves dos Santos Fernandes, Francisco José de Freitas}

\author{
Federal University of the State of Rio de Janeiro - UNIRIO, Rio de Janeiro, Brazil
}

\begin{abstract}
Background: In 2004, the deployment of Homeopathy in the pediatric ward at the University Hospital of Gaffrée Guinle - UNIRIO (HUGG at the Federal University of the State of Rio de Janeiro) was initiated in conjunction with both the Pediatric and Homeopathy Service. A research project approved by the HUGG Ethics and Research Committee was prepared to survey the most prevalent diseases. A team composed of medical students and doctors participating in the homeopathy course was formed and underwent training, enabling them to use the established protocols of action. A partnership was established with the Fluminense Federal University(Universidade Federal Fluminense - UFF), for the supply of drugs. In early 2009, the research project started, followed by homeopathic treatment in the pediatric ward.
\end{abstract}

Aim: To demonstrate the diseases and treatment using homeopathic therapy on patients in the pediatric ward at the HUGG-UNIRIO-Brazil.

Methodology: A sectional clinical study was carried out on patients participating in a research approved and registered by the Brazilian Research Ethics Committee, named,"The study of the effect of Homeopathic Treatment as an Adjunct Therapy on patients Hospitalized in the Pediatric Ward of HUGG". Criteria of Inclusion: Newborns up to the age of 16 of both sexes were admitted to the pediatric ward of HUGG, from May to October 2009. The diagnosis for admission being: respiratory, gastrointestinal and/or dermatologic diseases. A consent form had to be accepted and signed by the person responsible. Inclusion depended on the availability of having the appropriate homeopathic medicine in stock. Criteria of Exclusion: Cases of discontinuation of the homeopathic treatment or medical records not completed correctly. The medical records were analyzed individually. Microsoft Office Excel 2007 was used for data collection and analysis.

Results: 32 patients admitted: 80\% treated with Homeopathy; Diagnosis for admission: 73\% respiratory, 11.5\% dermatological, 4\% gastrointestinal and 11.5\% other diseases (malnutrition, adenomegaly, eyelid edema); 37\% had secondary diseases. An average of 4.7 medicaments (min 01-max09) was used per patient, 31\% repeated the medication in two different dinamizations. "Diagnosis for admission and medication used": a) respiratory: 79\% Pulmao histaminum, 32\% Antimonium tartaricum, 21\% Natrum sulfuricum, 21\% Sambucus nigra, 16\% Ipecacuanha, 16\% Nux vomica, 16\% Medorrhinum; b) dermatological: 100\% Apis mellifica, 33\% Dulcamara, 33\% Mezereum, 33\% Psorinum, 33\% Rhus toxicodendron, 33\% Alumina; c) gastrointestinal: 100\% Ipecacuanha, 50\% Gambogia, 50\% Alumina. Dinamizations used: 5, 6, 12,30 and $200 \mathrm{CH}$, and $200 \mathrm{FC}$. $55.5 \%$ of the medication used were only local effect medicines, $45.5 \%$ of the medication used were in association with the general effect medicines.

Conclusion: The need to use several homeopathic medicines was obseved. The practice of inpatients was shown to be similar to the emergency outpatient when using medicine for local effect and medicine for general effect. Due to the positive results obtained with homeopathy, patients not initially foreseen in the research were included in this study on demand from parents or persons responsible for the children. This 
study demonstrates the need for a greater number of patients to allow the creation of prescription protocols and case-control studies to identify the most effective homeopathic prescription techniques.

Keywords: Epidemiological studies; Homeopathic research; Pediatric Ward.

\section{References:}

Behrman RE, Jenson HB, Robert MN. Tratado de Pediatria. Editora Elsevier, 2002.

Demarque D, Jouanny J, Poitevin B, Saint-Jean, Y. Farmacologia e Matéria Médica Homeopática. Trad. Cláudio Roitman e Francisco José de Freitas. $1^{a}$ edição em português e $3^{a}$ edição francesa. Editora Organon, 2009 .

Fernandes DAS, Freitas FJ. Projetos, Formulários, Questionários e Fichas de Anamnese do Programa Homeopatia: Saúde e Qualidade de Vida. Biblioteca Nacional. Rio de Janeiro, 2009.

Freitas FJ. Apostila das Aulas do Prof. Francisco José de Freitas no Curso de Especialização Lato Sensu em Homeopatia da UNIRIO. Organizadora: Fernandes, DAS. Biblioteca Nacional. Rio de Janeiro, 2009.

\section{Perfil Clínico-Homeopático em Enfermaria de Pediatria de Hospital Universitário - Brasil}

\section{RESUMO}

Introdução: A implantação da Homeopatia na ala pediátrica do Hospital Universitário Gaffrée e Guinle HUGG da Universidade Federal do Estado do Rio de Janeiro - UNIRIO foi iniciada em 2004, com parcerias entre os serviços de Pediatria e Homeopatia. Foi preparado um projeto de pesquisa com as patologias doenças mais prevalentes e aprovado no Comitê de Ética e Pesquisa do Hospital. Uma equipe composta de médicos, estudantes de medicina e médicos em curso de homeopatia foi formada e treinada com os protocolos de ação estabelecidos. Uma parceria foi estabelecida com a Universidade Federal Fluminense - UFF para o fornecimento dos medicamentos. No início de 2009, o projeto de pesquisa começou e o tratamento na ala de pediatria foi iniciado.

Objetivos: Demonstrar o peril dos pacientes e medicamentos utilizados na terapêutica homeopática como tratamento co-adjuvante em pacientes da enfermaria pediátrica do HUGG-UNIRIO-Brasil.

Metodologia: Estudo clínico de coorte realizado com os pacientes da pesquisa longitudinal prospectiva observacional "Estudo sobre o efeito do tratamento homeopático como terapia adjuvante em pacientes internados na enfermaria pediátrica do HUGG", aprovada e registrada no Comitê de Ética e Pesquisa brasileiro. Critérios de inclusão: razão para admissão na enfermaria, sendo patologias respiratórias, gastrointestinais e/ou dermatológicas; termo de consentimento aceito; pacientes de ambos os sexos; recémnascidos até aos 16 anos de idade, internadas na enfermaria pediátrica do HUGG, de maio a outubro de 2009 motivo da internação tratado com medicamento homeopático adequado. Casos de interrupção do tratamento homeopático ou registros médicos não concluída corretamente foram excluídos. Os registros médicos foram analisados individualmente. Programa Microsoft Office Excel 2007 utilizado para a coleta e análise de dados. 
Resultados: 32 pacientes internados: $80 \%$ tratados com homeopatia; motivo da internação por patologias: $73 \%$ respiratórias, $11,5 \%$ dermatológicas, $4 \%$ gastrointestinais e 11,5\% outros (desnutrição, adenomegalia edema palpebral); 37\% possuiam patologias secundárias. Média de 4,7 medicamentos (min01-max09) por paciente, $31 \%$ repetidos medicamento(s), em duas dinamizações diferentes. Relação "Motivo da internação": "medicamento usado": a) respiratório: 79\% Pulmão histaminum, 32\% Antimonium tartaricum, 21\% Natrum sulfuricum, 21\% Sambucus nigra, 16\% Ipecacuanha, 16\% Nux vomica, 16\% Medorrhinum; b ) dermatológicos: 100\% Apis mellifica, 33\% Dulcamara, 33\% Mezereum, 33\% Psorinum, 33\% Rhus Toxicodendron, 33\% Alumina; c) gastrointestinal: 100\% Ipecacuanha, 50\% Gambogia, 50\% Alumina. Dinamizações utilizadas: 5, 6, 12,30 e $200 \mathrm{CH}$, e $200 \mathrm{FC} .55,5 \%$ de medicação de ação local apenas, $45,5 \%$ em associação com o medicamento de ação em geral.

Conclusão: Vários medicamentos homeopáticos foram usados. Uma prática semelhante em situações de emergência ambulatorial geralmente envolvendo medicamentos de ação local com medicamentos de ação geral; outros pacientes foram incluídos devido à solicitação de tratamento homeopático para outras doenças não previstas inicialmente na pesquisa e pelos relatórios de resposta clínica melhorada do paciente. Este estudo demonstra a necessidade de um maior número de pacientes para permitir a criação de protocolos de prescrição e estudos caso-controle para identificar as mais eficazes técnicas de prescrição homeopáticas.

Palavras-chave: Estudos epidemiológicos, pesquisa Homeopática; enfermaria pediátrica.

\section{(c)) BY-NC-ND Licensed to GIRI}

Support: UFF (FAU-UFF); Laboratório BOIRON Medicamentos Homeopáticos.

Conflict of interest: Authors had full access to all the data in this study and take complete responsibility for the integrity of the data and the accuracy of the data analysis.

Correspondence author: Débora Alves dos Santos Fernandes - deboraunirio@yahoo.com.br , Francisco José de Freitas fjdefreitas@gmail.com

How to cite this article: Fernandes DAS, Freitas FJ. Clinical-Homeopathic Profile in the Pediatric Ward at the University Hospital - Brazil. Int J High Dilution Res [online]. 2011 [cited YYYY Month dd]; 10(36): 206-208. Proceedings of the XXV GIRI Symposium and VIII CBFH; 2011 Sep 04-07; Foz do Iguaçu (Brazil). GIRI and ABFH; 2011; Available from: http://www.feg.unesp.br/ ojs/index.php/ijhdr/article/view/495/509 